\title{
Diagnosis of early myocarditis after respiratory or gastrointestinal tract viral infection: insights from cardiovascular magnetic resonance
}

\author{
Michael Jeserich • Stavros Konstantinides • \\ Manfred Olschewski • Gabor Pavlik • \\ Christoph Bode $\cdot$ Annette Geibel
}

Received: 1 December 2009/Accepted: 27 April 2010/Published online: 28 May 2010

(c) The Author(s) 2010. This article is published with open access at Springerlink.com

\begin{abstract}
Background The diagnosis of myocarditis continues to be a challenging task in clinical practice. The purpose of our study was to investigate cardiovascular magnetic resonance imaging in the diagnostic workup of ambulatory patients with the suspicion of early myocarditis after respiratory or gastrointestinal tract viral infection. The need for accurate diagnosis of early myocarditis arises from the low diagnostic accuracy of routine clinical tests.

Methods We examined 67 consecutive patients with symptoms of weakness, palpitations, and fatigue after respiratory or gastrointestinal tract infection. We compared these patients to 31 controls. ECG-triggered, T2-weighted, fast-spin-echo triple inversion recovery sequences and delayed enhancement imaging were obtained in all patients, as well as functional parameters of left ventricular
\end{abstract}

M. Jeserich · C. Bode $\cdot$ A. Geibel

Department of Cardiology and Angiology,

Albert Ludwig University of Freiburg,

Freiburg, Germany

M. Olschewski

Department of Medical Biometry and Statistics,

University of Freiburg, Freiburg, Germany

S. Konstantinides

Department of Cardiology and Pulmonary Medicine,

Georg August University of Goettingen,

Göettingen, Germany

G. Pavlik

Department of Health Sciences and Sports Medicine,

Faculty of Physical Education and Sports Sciences,

Semmelweis University, Budapest, Hungary

M. Jeserich $(\bowtie)$

Koenigstr. 39, 90402 Nuernberg, Germany

e-mail: info@Praxis-Jeserich.de function and dimensions. In addition, in 25 patients and 10 controls, ECG-triggered, T1-weighted, multi-slice spinecho images were obtained in axial orientation.

Results We found a significant difference between patients with suspected myocarditis and controls in T2-global myocardial signal intensity. In addition, the ratio of global myocardial signal intensity/muscle signal intensity was $2.3 \pm 0.4$ in patients and $1.8 \pm 0.3$ in controls, which was highly significant $(p<0.001)$. In 23 patients, a pathological late enhancement pattern was seen, but only in one of the controls. There was no significant difference in T1-signal parameters.

Conclusion Cardiovascular magnetic resonance technique is able to detect early myocardial involvement after respiratory or gastrointestinal tract infection.

Keywords Cardiovascular magnetic resonance imaging . Myocarditis - Triple inversion recovery sequence .

Respiratory and gastrointestinal tract viral infection .

T2-imaging $\cdot$ STIR

\author{
Abbreviations \\ CMR Cardiovascular magnetic resonance \\ ECG Electrocardiogram \\ STIR Spin-echo triple inversion recovery sequences \\ GD-DTPA Gadolinium-diethylenetriaminepentaacetate \\ LGE Late gadolinium enhancement
}

\section{Introduction}

Clinical presentations of myocarditis range from nonspecific systemic symptoms (fever, myalgias, palpitations, 
or exertional dyspnea) to fulminant hemodynamic collapse and sudden death. The extreme diversity of clinical manifestations has made the true incidence of myocarditis difficult to determine. Recent prospective postmortem data have implicated myocarditis in sudden cardiac death of young adults at rates of $8.6-12 \%[1,2]$. Furthermore, it has been identified as a cause of dilated cardiomyopathy in $9 \%$ of cases in a large prospective series [3]. Myocarditis is pathologically defined as "inflammation of the myocardium". However, despite this seemingly rather clear-cut definition, the diagnosis of myocarditis continues to be a challenging task in clinical practice.

During the past two decades, cardiovascular magnetic resonance (CMR) has evolved from a research tool to a clinically validated, safe and comprehensive imaging modality. It provides anatomic and functional information in acquired and congenital heart disease and is the most precise technique for quantification of ventricular volumes, function and mass. Delayed contrast enhancement is an accurate and robust method used in the diagnosis of ischemic, non-ischemic cardiomyopathies and myocarditis [4-8].

The diagnosis of myocardial involvement after viral infections is generally supported by the ECG (ST segment changes, arrhythmia, AV block), laboratory tests (troponin $\mathrm{T}$ or I, myoglobin, creatine kinase) and echocardiography (impairment of systolic or diastolic left ventricular function or increased wall thickness) [8, 9]. ECG abnormalities are usually transient and non-specific, whereas several patients have no electrocardiographic abnormalities [10-12]. In fact, some patients with a normal ECG and negative troponin or creatine kinase test may still have (suspected) myocarditis after or during a viral infection of the respiratory or gastrointestinal tract $[13,14]$.

On these grounds, the aims of the present study were (1) to examine ambulatory patients with suspicion of myocardial involvement after respiratory tract or gastrointestinal infection using CMR; (2) to assess different new CMR modalities in their practicability in decision-making. When compared with our pilot study [13], we examined a larger cohort and tested different modalities including T1 measurements and the late enhancement technique.

\section{Methods}

\section{Patients}

We prospectively examined 71 consecutive patients who were referred by general practitioners to an ambulatory cardiologic examination with suspicion of myocardial involvement after a viral infection. The patients showed symptoms of fatigue, weakness and/or palpitations or precordial discomfort after respiratory or gastrointestinal tract infections. Inclusion criteria were an infection during the last 12 weeks and ongoing symptoms. A CMR examination was performed. The results of these patients were compared with sex- and age-matched 31 healthy controls. All patients were carefully interviewed and examined by experienced cardiologists. Physical examination, ECG, blood pressure, laboratory testing, and echocardiographic evaluation were performed and a written informed consent was obtained from each subject.

One patient experienced claustrophobia during the CMR examination. In one patient, the image quality of the late enhancement was insufficient. In another, the image quality of the STIR sequence was not readable. One patient developed a contrast allergy after application of gadolinium. All these patients were excluded from analysis. Thus, the final cohort comprised 67 patients.

\section{Magnetic resonance imaging}

All images were acquired on a 1.5-T magnetic resonance system (Intera CV 1.5T, Philips, Best, The Netherlands) and specifically designed software (Release 11, Philips). The functional and morphological data were evaluated by the software view forum 6.5 (Philips). Regions of interest were drawn manually. Dedicated five element phase-array cardiac synergy coil was used for signal detection. We used constant level appearance techniques. Constant level appearance is a homogeneity correction applied to compensate for signal inhomogeneity attributable to the surface coils. It is equivalent to a SENSE acquisition with a SENSE factor of one to acquire the sensitivity maps for each synergy coil element (relative to the body coil sensitivity) that can be used to get a perfect uniformity correction. At the beginning of the pilot study, controls were scanned with cardiac and body coils. Signal homogeneity, stability, and robustness were better with the cardiac coil. To evaluate LV function and dimensions, we took 2, 3 and 4-chamber long-axis views and 3-D short axis volume data assessed by steady-state free precession imaging (field of view $350 \mathrm{~mm}$, matrix $256 \times 256$, echo time $1.6 \mathrm{~ms}$, repetition time $4.0 \mathrm{~ms}$, flip angle $60^{\circ}$, slice thickness $10 \mathrm{~mm}$, no gap). In all patients, phase-contrast velocity images in the aorta ascendens were obtained to calculate stroke volume and to rule out a significant aortic insufficiency (field of view $300 \mathrm{~mm}$, matrix $256 \times 256$, echo time $3.7 \mathrm{~ms}$, repetitions time $6.1 \mathrm{~ms}$, flip angle $25^{\circ}$, slice thickness $8 \mathrm{~mm})$.

Diagnosis of myocardial inflammation

ECG-triggered, T2-weighted, fast-spin-echo triple inversion recovery sequences (STIR) were performed in all 
patients and controls in a short axis view covering the whole left ventricle as previously described [13]. The relative myocardial signal intensity was calculated by the ratio of myocardial signal intensity and muscle signal intensity [15]. The homogeneity of the signal intensity of the region of interest has been studied intensively at the start of the pilot study in volunteers and patients. A very good robustness of the performed STIR sequence showing a homogeneous signal at different time points in the same volunteers was found.

In addition, in 25 patients and 10 controls, ECG-triggered, T1-weighted, multi slice spin-echo images were obtained in axial orientation (3-4 acquisitions, field of view $370 \mathrm{~mm}$, matrix size $256 \times 256$, slice thickness $10 \mathrm{~mm}$, no gap, echo time $20 \mathrm{~ms}$, flip angle $90^{\circ}$, relaxation time 480-725 ms) with identical parameters before and in 21 patients and 9 controls after intravenous bolus of $0.1 \mathrm{mmol} / \mathrm{kg}$ gadolinium-diethylenetriaminepentaacetate (Gd-DTPA) (Magnevist, Schering AG, Germany) (early phase, 1-2 min after bolus injection) A saturation slice across the atria was positioned to reduce signals from slowflowing blood into the left ventricle. The signal intensity in the myocardial wall and in the skeletal muscles was measured; 5-10 regions of interest were drawn into the septum, anterior, and lateral wall before and after application of Gd-DTPA. In addition, 5-10 regions of interests were drawn into the skeletal muscles (erector spinae muscle or lattissimus dorsi) with homogenous signal. The relative myocardial signal intensity was calculated by the following formula: intensity after Gd-DTPA minus the intensity before Gd-DTPA divided by the intensity before Gd-DTPA ratio of myocardial signal intensity and muscle signal intensity [15].

\section{Delayed enhancement}

Delayed enhancement imaging was obtained 10 min after a second bolus of $0.1 \mathrm{mmol} / \mathrm{kg}$ intravenous gadolinium by hand injection. 3D inversion recovery turbo gradient echo sequence ( 2 acquisitions, field of view $330 \mathrm{~mm}$, matrix size $256 \times 256$, slice thickness $5 \mathrm{~mm}$, no gap, echo time $1,4 \mathrm{~ms}$, TR shortest, flip angle $15^{\circ}$, an inversion time 230 $280 \mathrm{~ms}$ ) was optimized for each measurement for maximal myocardial signal suppression. 3D volume of the left ventricle was obtained covering the complete left ventricle without gap during two breath holds.

\section{Statistical analysis}

Data are presented as mean and standard deviation for quantitative variables and as absolute and relative frequencies for categorical variables. For the evaluation of diagnostic parameters, such as specificity, STIR was dichotomised at its optimal value. Comparisons of variables between patients and controls were performed by means of Wilcoxon's rank tests. All tests were two-sided and used a significance level of 0.05 to indicate statistical significance. A percent segmental agreement between STIR values and LGE was obtained in the 23 patients who presented with positive LGE.

\section{Results}

Patient characteristics

Patient baseline characteristics are shown in Table 1. Only four patients had to be admitted to the hospital, one female patient with a rapidly deteriorating LV function with a minimal ejection fraction of $20 \%$ and one patient with additional pericardial effusion. Two other patients were admitted for chest pain to exclude an acute coronary syndrome. In two patients, a biopsy was performed and parvo B 19 virus was detected. All the other patients were dealt with as outpatients. In several patients documented ventricular or atrial premature beats remained over time, whereas the other ones completely recovered. The patients were followed as outpatients for up to 5 years (6-60 months, median $32.9 \pm 13.4$ months). One patient is developing dilatative cardiomyopathy, left ventricular function actually is $49 \%$, LVEDD $62 \mathrm{~mm}$, in another patient function remains $50 \%$ with normal diameters. LV function is normal in the remaining patients $(>50 \%)$. In the acute phase, the symptomatic patients with ventricular premature beats were treated with potassium, magnesium

Table 1 Clinical characteristics of the 67 patients

\begin{tabular}{lc}
\hline Variable $(\%)$ & Patients \\
\hline Male sex & $45(67)$ \\
Weakness & $50(75)$ \\
Fatigue & $44(66)$ \\
Dyspnea & $26(39)$ \\
Palpitations & $22(33)$ \\
Chest pain & $5(7)$ \\
Ventricular premature beats & $13(19)$ \\
Atrial premature beats & $6(9)$ \\
New onset atrial fibrillation & $4(6)$ \\
Left bundle branch or hemi-block & $5(7)$ \\
New ST/T-changes & $5(7)$ \\
Small pericardial effusion & $8(12)$ \\
\hline
\end{tabular}

One patient had a new sinoatrial exit block, one patient a new right deviation of the electrical position of the heart. Some patients had more than one symptom 
and low-dose beta-blockers on an individual basis. Patients with severe exertional dyspnea and borderline ejection fraction were treated in addition with ACE inhibitors. All patients were advised to avoid any physical activities during the acute phase.

\section{Functional parameters}

The values are listed in Table 2. Left ventricular ejection fraction was slightly lower in patients versus controls. No control had a left ventricular function below $55 \%$, whereas six patients had a function of $55 \%$ or less (minimal $52 \%$ ). There were no significant differences in stroke volume, cardiac output, left ventricular end-diastolic volume, and left ventricular end-diastolic diameter between patients and controls.

Diagnosis of inflammation

\section{STIR (T2-weighted imaging)}

We found a significant difference between patients with suspected myocarditis and controls in global myocardial signal intensity, in the signal intensity of the septum, anterior, lateral, and inferior wall. The ratios of septal, anterior, lateral, and inferior myocardial signal intensity/ muscle signal intensity were also significantly higher in patients as compared to controls (Table 3). Figures 1 and 2 depict examples of two patients. The signal of the skeletal muscle was very similar in patients and controls.

The best cutoff value to differentiate patients and controls was 2.14 (global myocardial signal intensity/muscle signal intensity). The sensitivity was $74.5 \%$, the specificity $93.5 \%$, the positive-predictive value $96.2 \%$, and the negative-predictive value $63.0 \%$, and the diagnostic accuracy $80.6 \%$.

We performed a percent segmental agreement and found a correlation regarding the ratios of septal, anterior, lateral, and inferior myocardial signal intensity/muscle signal intensity and a positive late enhancement in the corresponding area in 17 of 23 cases (74\%). The myocardial signal intensity of the septum, anterior, lateral, and inferior wall corresponded in 13 of 23 cases $(57 \%)$.

\section{Contrast media-enhanced T1-weighted images}

In the first 25 patients and first 10 controls, the myocardial signal intensity before contrast application was $603 \pm 162$ in patients and $639 \pm 188$ in controls, after contrast application $913 \pm 163$ and $965 \pm 160$, n.s. The ratio of myocardial and muscle signal intensity was $1.4 \pm 0.3$ in patients and $1.8 \pm 0.4$ in controls, n.s., before, and was $2.1 \pm 0.6$ versus $1.8 \pm 0.4$ after gadolinium, n.s. The relative enhancement had a range from +43 to -4 in patients and from +4 to -107 in controls and was not interpretable. Therefore, we stopped the $\mathrm{T} 1$ approach after the first 25 patients and 10 controls.

\section{Delayed gadolinium-enhanced imaging}

Contrast enhancement was present in 23 of 67 patients (34\%), $p=0.001$ versus controls. Regions of contrast enhancement were usually seen in a patchy distribution originating primarily from the epicardial quartile or midmyocardial location of the wall with one or several foci within the myocardium and were most frequently located in the septum and in the lateral free wall (14 patients) (Figs. 3, 4). Ten of these patients had a septal and lateral location. One patient had a location in the inferior wall and three patients had a diffuse enhanced myocardium. Contrast enhancement was never found originating from the subendocardial portion of the wall, as would be typical for myocardial infarction. In the control group, only one patient had a small lateral mid-myocardial enhancement.

Patients with fatigue and weakness after respiratory infection and no cardiac involvement

Nine of the 67 patients had mild symptoms with fatigue and weakness after a respiratory infection, but no new palpitations and no new documented premature beats or

Table 2 Magnetic resonance functional measurements in control subjects and patients

\begin{tabular}{lccc}
\hline Variable & Patients $(n=67)$ & Controls $(n=31)$ & $p$ value \\
\hline Mean age [years (SD)] & $50.1 \pm 14.8$ & $53.9 \pm 15.7$ & 0.18 \\
Ejection fraction (\%) & $60.0 \pm 5.5$ & $62.8 \pm 5.3$ & 0.02 \\
Stroke volume (mL) & $91.5 \pm 20.6$ & $95.3 \pm 21.8$ & 0.67 \\
LV end-diastolic volume (mL) & $154.0 \pm 33.3$ & $150.0 \pm 36.6$ & 0.38 \\
LV end-diastolic diameter (mm) & $52.3 \pm 4.8$ & $51.9 \pm 4.1$ & 0.50 \\
Cardiac output (L/min) & $6.2 \pm 1.1$ & $6.8 \pm 1.7$ & 0.13 \\
\hline
\end{tabular}

Values are expressed as mean \pm standard deviation

$L V$ left ventricular 
Table 3 Magnetic resonance measurements of inflammation in control subjects and patients

\begin{tabular}{lccc}
\hline Variable & Patients $(n=67)$ & Controls $(n=31)$ & $p$ value \\
\hline STIR of the left ventricular myocardium & $668 \pm 288$ & $526 \pm 91$ & $<.001$ \\
STIR of the skeletal muscle & $288 \pm 51$ & $294 \pm 62$ & 0.55 \\
STIR myocardium/skeletal muscle & $2.3 \pm 0.4$ & $1.8 \pm 0.3$ & $<46 \pm 100$ \\
STIR of the septum & $686 \pm 135$ & $1.9 \pm 0.3$ & $<.001$ \\
STIR septum/skeletal muscle & $2.4 \pm 0.4$ & $498 \pm 99$ & $<.001$ \\
STIR of the anterior wall & $648 \pm 157$ & $1.7 \pm 0.3$ & $<15 \pm 97$ \\
STIR anterior wall/skeletal muscle & $2.3 \pm 0.4$ & $1.8 \pm 0.3$ & $<0.001$ \\
STIR of the lateral wall & $679 \pm 140$ & $<0.001$ \\
STIR lateral wall/skeletal muscle & $2.4 \pm 0.4$ & $<0.001$ \\
\hline
\end{tabular}

Values are expressed as mean \pm standard deviation

$S T I R$ T2-weighted, fast-spin-echo triple inversion recovery sequences

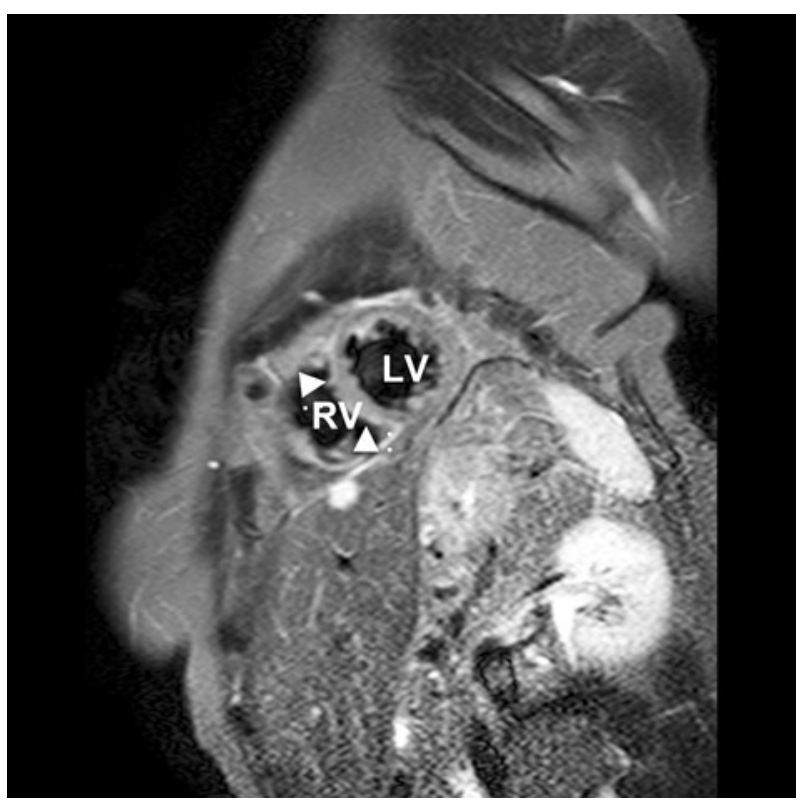

Fig. 1 An example of T2-weighted (STIR) image of a patient revealing enhanced signal intensity. This provides evidence of myocardial edema. $L V$ left ventricle, $R V$ right ventricle. Filled triangle elevated signal intensity of the myocardium

other rhythm disturbances. Thus, on a clinical basis, an overt myocarditis would be less likely. The mean age was 52 years, male sex 7 (78\%), global myocardial signal intensity was $553 \pm 139$ and the ratio of global myocardial signal intensity/muscle signal intensity was $2.1 \pm 0.3$, thus in the range between patients and controls.

\section{Discussion}

We found a high prevalence of myocardial inflammation in patients with symptoms of fatigue, weakness, and/or palpitations after respiratory tract or gastrointestinal infection. In addition, one-third of patients had a mid-myocardial or

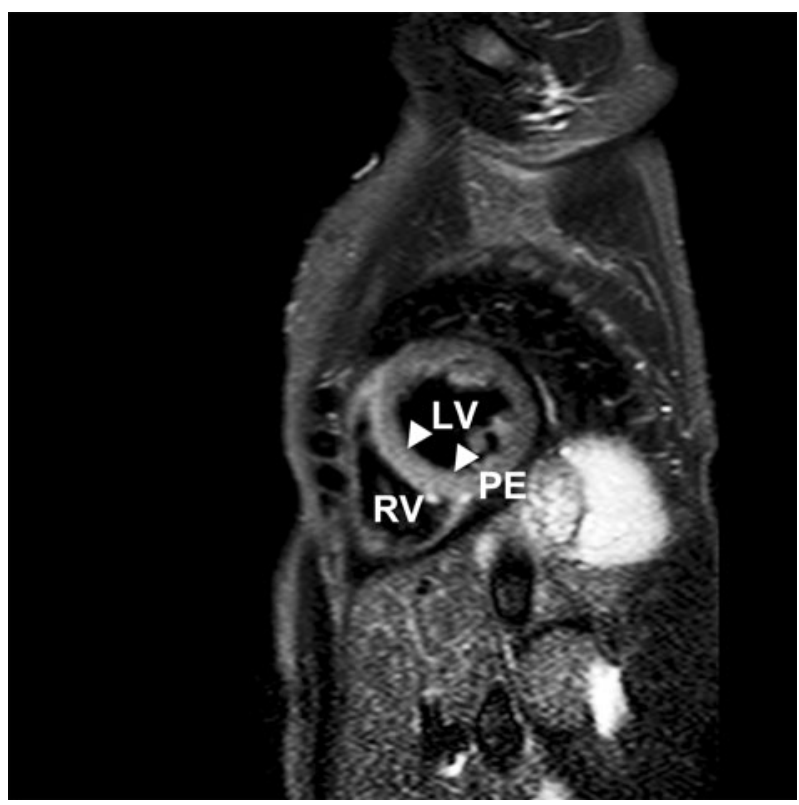

Fig. 2 Another example of T2-weighted (STIR) image of a patient showing an enhanced signal intensity of the septal wall (filled triangle). $L V$ left ventricle, $R V$ right ventricle, $P E$ pericardial effusion

subepicardial late gadolinium enhancement indicating fibrosis of non-ischemic injury. Thus, CMR is able to make a positive diagnosis of myocarditis and add substantial diagnostic information to the standard approach with ECG, laboratory values and echocardiography.

The need for accurate diagnosis of early myocarditis arises from the low diagnostic accuracy of routine clinical tests. Even a negative troponin test and absence of ST elevation or depression cannot rule out an early form of myocarditis [8, 14]. In our study, we found that patients with suspected myocarditis had a highly significant elevated signal intensity of the myocardium as compared to controls. This indicates edematous tissue that may reflect cardiac involvement as the first step of myocarditis. 


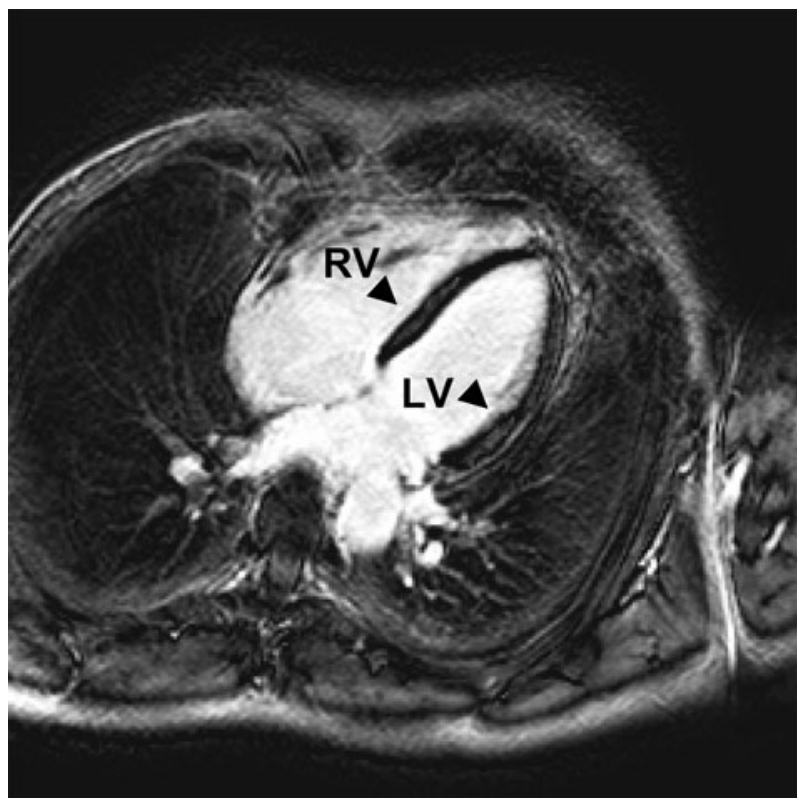

Fig. 3 An example of a late enhancement pattern of a patient illustrating contrast enhancement of the septum and lateral free wall (filled triangle). Of notation is the mid-myocardial location. $L V$ left ventricle, $R V$ right ventricle

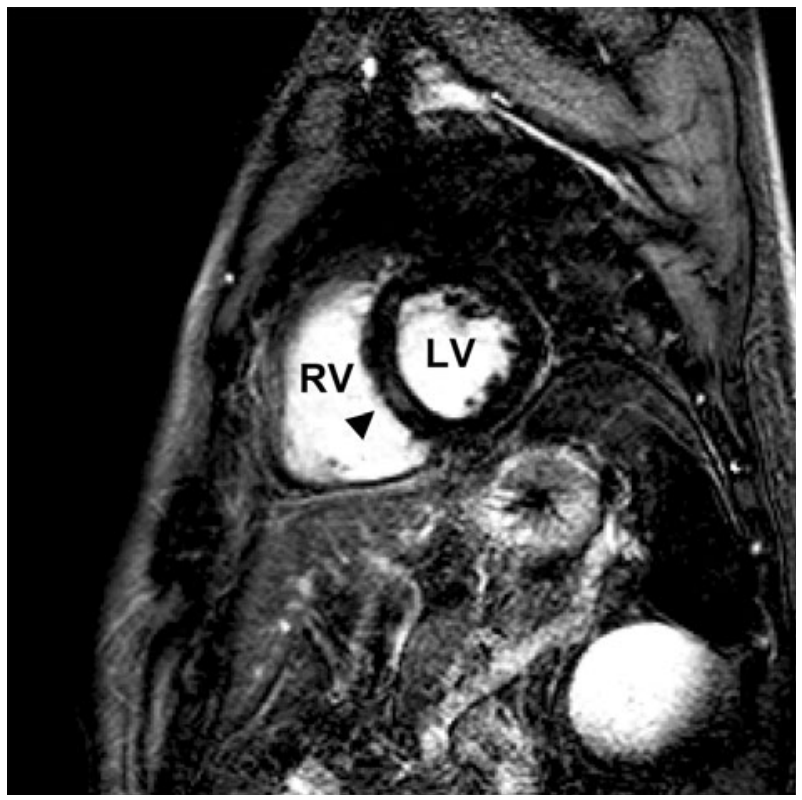

Fig. 4 Another example of a late gadolinium enhancement pattern of a patient showing contrast enhancement of the septum, mid-myocardial location (filled triangle). Two chamber view; $L V$ left ventricle, $R V$ right ventricle

\section{Myocardial inflammation}

Cardiovascular magnetic resonance is able to provide detailed information on myocardial tissue characteristics. Tissue hyperemia is an integral component of the acute inflammatory reaction in the myocardium and can be detected by T2-weighted imaging. This sequence has been clinically used to detect edema in acute infarction, sarcoidosis, and acute rejection following cardiac transplantation [16-18].

In patients with myocarditis, case reports have reported the use of conventional T2-weighted spin echos [19-22]. New developments, especially a triple inversion breath hold sequence with short acquisition time (STIR) have led to a much better image quality. These sequences had been tested in previous studies [13, 23-25]. The highly significant differences in myocardial enhancement between patients and controls found by this new T2-modality in our study confirm that CMR is able to diagnose myocardial involvement in patients with viral infections.

Sensitivity, specificity, positive predictive value, negative predictive value and accuracy of a T2-weighted image were obtained in four previous studies: a higher sensitivity as compared to our study was found in the study by Rieker [26] and Abdel-Aty et al. [23], a higher specificity in the study of Laissy et al. [27], the accuracy was comparable to our findings in the study of Abdel-Aty et al. [23], positive predictive value was higher in the study of Laissy et al. [27], but lower in the others, whereas the negative predictive value was comparable to the study of Gutberlet et al. [25], and higher in the other studies.

The group of Friedrich and Schulz-Menger et al. [15, 23] examined T1-weighted images, especially contrast media-enhanced in comparison to healthy volunteers. They found an increased absolute and relative enhancement compared with skeletal muscle. These results were confirmed by another group [25]. Gd-DTPA is an approved extracellular T1-enhancing contrast agent [28] which increases membrane permeability or increases capillary blood flow resulting in an increase in volume of distribution. In our study, we found no significant differences in the absolute or relative T1-weighted images before or after contrast application. In addition, we found it difficult to establish stable conditions after the application of $\mathrm{Gd}$ DTPA. There is a continuous change in the zero run through after the application of the contrast media, thus precluding stable conditions.

A difference between our study and the former results may be the course and extent of myocarditis. We examined ambulatory patients with normal or only slightly reduced left ventricular function. In previous studies, the patients were more seriously sick and needed hospitalization. Thus, for the very early course of myocarditis, a contrastenhanced T1-approach may not be a useful tool.

Late gadolinium enhancement pattern

Normal myocardium does not enhance because extracellular magnetic resonance contrast agents, such as Gd-DTPA, 
are excluded from the myocyte intracellular space by intact sarcolemmal membranes and also, little interstitial space is available between densely packed myocytes [7]. This technique has been shown to be effective in identifying the presence, location, and extent of acute and chronic myocardial infarction [5-7, 29, 30] and other cardiac diseases [31-33]. Late gadolinium enhancement has been investigated in controlled studies with patients suspected of having myocarditis [23-25, 34, 35]. Images acquired late (10-20 min) after application of paramagnetic contrast agents provide a sensitive tool for detecting myocardial fibrosis or necrosis, which is distinguished by bright lateenhancement regions due to the increased volume of distribution and delayed washout of the contrast agent from affected myocardium [36]. The major difference to infarcted regions is that areas of tissue damage within a myocardiac focus are usually smaller than are areas of infarcts and the former are dispersed in a patchy pattern throughout the focus [36, 37].

In our study, all patients had subepicardial or centromyocardial location of contrast enhancement and the preferred locations were lateral and septal. The regions of contrast enhancement revealed a patchy pattern typical of non-ischemic injury. The distribution ranged from one to several foci, and from almost normal to a diffusely enhanced myocardium. No patient had a subendocardial location or a location according to territorial distribution of epicardial vessels. Thus, this study supports the notion that detection of a subepicardial or centromyocardial scar is highly suggestive of myocarditis in suspected patients. In addition, we found that the majority of patients had lateenhancement regions in the septal and lateral wall. Other authors $[24,34,38]$ found it predominantly lateral, followed by a septal location.

We conclude that a subepicardial or centromyocardial location of contrast enhancement in the septum or lateral wall within a clinical context suspicious of myocarditis provides evidence of myocardial involvement.

\section{Study limitations and clinical implications}

A major limitation of our study is that biopsies were performed only in two patients. Thus, we have no independent proof that our patients did indeed suffer from myocarditis. Myocarditis can be diagnosed with certainty only by endomyocardial biopsy, which must be examined by histological, immunohistological and molecular techniques to obtain maximal sensitivity. However, optimal diagnosis of a biopsy is subject to the problem of sampling error resulting in a relatively low sensitivity. Furthermore, myocardial biopsy is in practice used only rarely, despite a low complication rate [39]. Thus, the diagnosis of myocarditis is often based merely on suspicion. In our study, there were clinical features, such as new palpitations, new exertional dyspnoea, fatigue, chest pain, and documented ventricular or atrial premature beats as well as branch bundle block and other ECG changes that supported the clinical suspicion of myocarditis. We used a special modern CMR sequence of inflammation (STIR) which has been used to detect cardiac inflammation in previous studies [23-25, 40], and is recommended in a recent consensus paper [41]. Thus, we have strong evidence of myocardial involvement in our patient sample.

We found that the cutoff value of 2.14 had a relatively high specificity (ratio of heart to skeletal muscle) in T2weighted-STIR images, but a comparable or even lower sensitivity as compared to others [24, 26-28] which may be due to different study population and CMR modalities.

In conclusion, in patients with suspected myocarditis, CMR tomography is helpful in decision-making. With this technique, it is possible to analyze inflammation, edema and necrosis in addition to functional parameters, such as left ventricular function, regional wall motion and dimensions. We were able to identify patients with cardiac involvement after respiratory or gastrointestinal tract viral infection. Whereas myocarditis may lead to heart failure this early detection ability may be a promising tool.

Acknowledgments We thank Petra Nuß for collecting the data and typing parts of the manuscript. We thank Lewis and Thea Hunisch for revising the manuscript.

\section{Conflict of interest statement None.}

Open Access This article is distributed under the terms of the Creative Commons Attribution Noncommercial License which permits any noncommercial use, distribution, and reproduction in any medium, provided the original author(s) and source are credited.

\section{References}

1. Fabre A, Sheppard MN (2006) Sudden adult death syndrome and other non-ischaemic causes of sudden cardiac death: a UK experience. Heart 92:316-320

2. Doolan A, Langlois N, Semsarian C (2004) Causes of sudden cardiac death in young Australians. Med J Aust 180:110-112

3. Felker GM, Hu W, Hare JM, Hruban RH, Baughman KL, Kasper EK (1999) The spectrum of dilated cardiomyopathy: the Johns Hopkins experience with 1, 278 patients. Medicine (Baltimore) 78:270-283

4. Marcu CB, Beek AM, van Rossum AC (2006) Clinical applications of cardiovascular magnetic resonance imaging. CMAJ 175:911-917 (review)

5. Kim RJ, Fieno DS, Parrish TB et al (1999) Relationship of MRI delayed contrast enhancement to irreversible injury, infarct age, and contractile function. Circulation 100:1992-2002

6. Jensen CJ, Bleckmann D, Eberle HC et al (2009) A simple MR algorithm for estimation of myocardial salvage following acute ST segment elevation myocardial infarction. Clin Res Cardiol 98:651-656 
7. Mahrholdt H, Wagner A, Judd RM, Sechtem U (2002) Assessment of myocardial viability by cardiovascular magnetic resonance imaging. Eur Heart J 23:602-619

8. Mangin M, Marhold H, Sechtem U (2006) Diagnosis of myocarditis: description and assessment of available methods. Dtsch Med Wochenschr 131:1228-1234

9. Skouri HN, Dec GW, Friedrich MG, Cooper LT (2006) Noninvasive imaging in myocarditis. J Am Coll Cardiol 48:2085-2093

10. Eckart RE, Love SS, Atwood JE et al (2004) Incidence and follow-up of inflammatory cardiac complications after smallpox vaccination. J Am Coll Cardiol 44:201-205

11. Marnach M, VanWinter J, Watson W (2007) Myocarditis: an unusual cause of postpartum fever in pregnancy complicated by antiphospholipid syndrome. Am J Perinatol 24:405-408

12. Kontogianni DD, Kouris NT, Papoulia EP, Goranitou GS, Grassos HA, Babalis DK (2007) Discordance between echocardiographic and MRI findings in two cases of acute myocarditis mimicking myocardial infarction. Int J Cardiol 114:e21-e23

13. Jeserich M, Olschewski M, Th Bley et al (2009) Cardiac involvement after respiratory tract viral infection-detection by cardiac magnetic resonance. J Comput Assist Tomogr 33:15-19

14. Carniel E, Sinagra G, Bussani R et al (2004) Fatal myocarditis: morphologic and clinical features. Ital Heart J 5:702-706

15. Friedrich MG, Strom O, Schulz-Menger J, Marcinirk H, Luft FC, Dietz R (1998) Contrast media-enhanced magnetic resonance imaging visualizes myocardial changes in the course of viral myocarditis. Circulation 97:1802-1809

16. Aletras AH, Tilak GS, Natanzon A et al (2006) Retrospective determination of the area at risk for reperfused acute myocardial infarction with $\mathrm{T} 2$-weighted cardiac magnetic resonance imaging: histopathological and displacement encoding with stimulated echoes (DENSE) functional validations. Circulation 113:1865-1870

17. Vignaux O, Dhote R, Duboc D et al (2002) Clinical significance of myocardial magnetic resonance abnormalities in patients with sarcoidosis: a 1-year follow-up study. Chest 122:1895-1901

18. Marie PY, Angioi M, Carteaux JP et al (2001) Detection and prediction of acute heart transplant rejection with the myocardial $\mathrm{T} 2$ determination provided by a black-blood magnetic resonance imaging sequence. J Am Coll Cardiol 37:825-831

19. Gagliardi MG, Bevilacqua M, Di Renzi P, Picardo S, Passariello R, Marcelletti C (1991) Usefulness of magnetic resonance imaging for diagnosis of acute myocarditis in infants and children: an comparison with endomyocardial biopsy. Am J Cardiol 68:1089-1091

20. Gagliardi MG, Polletta B, Di Renzi P (1999) MRI for the diagnosis: an flow-up of myocarditis. Circulation 99:458-459

21. Bergler-Klein J, Sochor H, Stanek G, Globits S, Ullerch R, Glogar D (1993) Ingium 111-monoclonal antimyosin antibody and magnetic resonance imaging in the diagnosis of acute lyme myopericarditis. Arch Inter Med 153:2696-2770

22. Shen CT, Jeng CM, Lin YM, Chieng PU (1993) Intensification of relative myocardial $\mathrm{T} 2$-weighted magnetic resonance signals in patients with acute viral myocarditis (report of one CASE). Acta Paediatr Sin 34:405-411

23. Abdel-Aty H, Boyé P, Zagrosek A et al (2005) Diagnostic performance of cardiovascular magnetic resonance in patients with suspected acute myocarditis: comparison of different approaches. J Am Coll Cardiol 45:1815-1822

24. Assomull RG, Lyne JC, Keenan N et al (2007) The role of cardiovascular magnetic resonance in patients presenting with chest pain, raised troponin and unobstructed coronary arteries. Eur Heart J 28:1242-1249

25. Gutberlet M, Spors B, Thoma T et al (2008) Suspected chronic myocarditis at cardiac MR: diagnostic accuracy and association with immunohistologically detected inflammation and viral persistence. Radiology 246:401-409

26. Rieker O, Mohrs O, Oberholzer K, Kreitner KF, Thelen M (2002) Cardiac MRI in suspected myocarditis. Rofo Fortschr Geb Rontgenstr Neuen Bildgeb Verfahr 174:1530-1536

27. Laissy JP, Messin B, Varenne O et al (2002) MRI of acute myocarditis: a comprehensive approach based on various imaging sequences. Chest 122:1638-1648

28. Liu PP, Yan AT (2005) Cardiovascular magnetic resonance for the diagnosis of acute myocarditis. J Am Coll Card 45:18231835

29. Neizel M, Futterer S, Steen H et al (2009) Predicting microvascular obstruction with cardiac troponin $\mathrm{T}$ after acute myocardial infarction: a correlative study with contrast-enhanced magnetic resonance imaging. Clin Res Cardiol 98:555-562

30. Pilz G, Bernhardt P, Harrer E, Klos M, Höfling B (2008) Cardiac magnetic resonance imaging: an essential diagnostic aid in suspected myocardial reinfarction in a 40 year old woman. Clin Res Cardiol 97:280-283

31. Koeth O, Mark B, Kilkowski A et al (2008) Clinical, angiographic and cardiovascular magnetic resonance findings in consecutive patients with Takotsubo cardiomyopathy. Clin Res Cardiol 98:66-70

32. Eitel I, Fuernau G, Walther C et al (2008) Delayed enhancement magnetic resonance findings in connective patients with Takotsubo cardiomyopathy. Clin Res Cardiol 97:623-627

33. Cowan BR, Young AA, Anderson C et al (2009) The cardiac MRI substudy to ongoing telmisartan alone and in combination with ramipril global endpoint trial/telmisartan randomized assessment study in ACE-intolerant subjects with cardiovascular disease: analysis protocol and baseline characteristics. Clin Res Cardiol 98:421-433

34. Mahrholdt H, Wagner A, Deluigi CC et al (2006) Presentation, patterns of myocardial damage, and clinical course of viral myocarditis. Circulation 114:1581-1590

35. Laissy JP, Hyafil F, Feldman LJ et al (2005) Differentiating acute myocardial infarction from myocarditis: diagnostic value of early- and delayed-perfusion cardiac MR imaging. Radiology 237:75-82

36. Babu-Narayan SV, McCarthy Karen P et al (2007) Images in cardiovascular medicine. Myocarditis and sudden cardiac death in the young extensive fibrosis suggested by cardiovascular magnetic resonance in vivo and confirmed post mortem. Circulation 166e: $122-125$

37. Hombach V, Merkle N, Kestler HA et al (2008) Characterization of patients with acute chest pain using cardiac magnetic resonance imaging. Clin Res Cardiol 97:760-767

38. Mahrholdt H, Goedecke C, Wagner A et al (2004) Cardiovascular magnetic resonance assessment of human myocarditis: a comparison to histology and molecular pathology. Circulation 109:1250-1258

39. Holzmann M, Nicko A, Kühl U et al (2008) Complication rate of right ventricular endomyocardial biopsy via the femoral approach: a retrospective and prospective study analyzing 3048 diagnostic procedures over an 11-year period. Circulation 118:1722-1728

40. Zagrosek A, Abdel-Aty H, Boyé P et al (2009) Cardiac magnetic resonance monitors reversible and irreversible myocardial injury in myocarditis. JACC Cardiovasc Imaging 2:131-138

41. Friedrich MG, Sechtem U, Schulz-Menger J et al (2009) Cardiovascular magnetic resonance in myocarditis: a JACC White Paper. J Am Coll Cardiol 53:1475-1487 